\title{
EDITORIAL
}

\section{World leaders describe the latest in IGF research}

\author{
Emily Jane Gallagher and Derek LeRoith \\ Endocrinology, Diabetes and Bone Diseases, Icahn School of Medicine at Mount Sinai, New York, New York, USA \\ Correspondence should be addressed to D LeRoith: derek.leroith@mssm.edu
}

One of the most pervasive systems in biology is the insulinlike growth factor (IGF) system of ligands, binding proteins and receptors. Since their discovery in the 1950s, the interest in the IGFs has motivated biologists, biochemists, molecular geneticists, evolutionists, physiologist, pharmacologists and pharmaceutical and biotech companies. The IGF system plays important roles in normal physiology but in addition has been shown to be intimately involved in a wide array of disease processes including growth retardation, diabetes, cancer and neurological disorders, to name but a few. Thus, there has been interest in stimulating the IGF system on the one hand and inhibiting the system on the other hand. The current issue has been created on a range of topics that cover some of the recent developments in the field to give the reader a taste of this exciting and relevant biological system.

As with most physiological systems, it behooves investigators to understand the underlying processes involved in the actions of the IGF system.

In the opening review (Hakuno \& Takahashi 2018), the authors describe the activation of the IGF1 receptor (IGF1R) - a receptor that is expressed by all cells and tissues except the hepatocyte. Following ligand binding and autophosphorylation of the receptor, several substrates are bound and activated, and in turn activate signaling pathways including, for example, the phosphatidylinositol 3-kinase and MAPK pathways. These and other pathways induce cell proliferation, cell differentiation and cell survival. Thus, the IGF/IGF1R is quite ubiquitous and affects most cells in the body. The authors also describe their most recent findings regarding the maintenance of prolonged action of IGFs by certain cellular events allowing the sustained function necessary where growth is required.
While the cellular responses of the IGF1R follow the binding of IGF1 and/or IGF2 to the receptor, in normal physiology, there are six IGF-binding proteins that regulate these interactions. In the review by Bach (2018), the normal and pathological effects of the six IGF-binding proteins (IGFBPs) on the ligand-receptor interactions are described. In addition, the ligand-independent effects of the IGFBPs on cellular function at the cell surface and intracellularly are discussed. The importance of the IGFBPs has become well recognized through in vitro cellular studies as well as genetically engineered mouse models.

The IGF system has long being known as mediating the effects of growth hormone $(\mathrm{GH})$ on longitudinal growth. GH-deficient people and animal models have low circulating IGF1 levels, whereas GH excess, which occurs in gigantism and acromegaly, is associated with elevated circulating IGF1 levels. Recent studies have delved into the actual mechanisms of action of the IGFs on bone.

Three reviews cover the skeletal effects of IGFs. Yakar et al. (2018) describe their excellent studies using genetically engineered mouse models to study the effect of circulating IGFs and IGFBPs on skeletal function. Using the liver-specific IGF1, IGFBP3 and acid-labile subunit knockout mice, among other models, they explain the role of circulating IGF1 on bone metabolism. Lindsey et al. (2018) describe the recent findings on the interaction of IGF1 and ephrin signaling pathways. These exciting new findings are being developed to provide a greater understanding of the metabolic changes that could explain disorders such as osteoporosis and fractures. An exciting new area that has captured the imagination of researchers in every field of biology and medicine is the role of the microbiome in modulating many different 
diseases. Poinsot et al. (2018) have focused on the effects of the microbiome on nutrient status and how the interaction with the IGF system affects bone growth. They review the various animal models that support this contention, while fully recognizing the fact that no clinical evidence is as yet available to confirm these effects in humans. They point out that clinical studies will be required to understand the role of the microbiome role in human disease states.

Whole body metabolism is an area of physiology that was previously attributed almost exclusively to insulin and the insulin receptor signaling. However, studies have shown conclusively that the IGF system is an important factor in normal and abnormal metabolism. In the review by Kineman et al. (2018), they describe the results of numerous genetically engineered mouse models involving the GH/IGF1/insulin genes and their receptors. These models commonly distort glucose and lipid metabolism, for example resulting in insulin resistance and hepatic lipid accumulation. While these metabolic changes are not always directly induced by IGF1, they are often indirect sequelae to the effects of IGF1 on metabolism.

In a separate review, Clemmons (2018) describes the interactions of the various IGFBPs with the ligands, IGF1 and IGF2 and how these may affect the interactions of the ligands with their receptors. Both the IGFBPs and the ligands are affected by changes in metabolism and are regulated by other factors. The responses of the target tissues to IGFs and IGFBPs are therefore affected by these factors. Thus, the IGFBPs in addition to the ligands play a critical role in the response to metabolism and in the regulation metabolic processes.

Following the demonstration that circulating IGF1, and possibly IGFBP3 levels, correlated with increased risk of cancer, studies in cancer cells and mouse models demonstrated that lower IGF1 levels reduced tumorigenesis and metastases. Furthermore, the finding that cancer cells had increased IGF1R expression led many investigators to study the mechanisms that may explain the link between IGF1R and tumor progression. The review by Mancarella \& Scotlandi (2018) describes Dr Scotlandi's interesting work on the IGF system in sarcoma pathogenesis. Given, that Ewing sarcomas were shown uniquely to respond to anti-IGF1R monoclonal antibody therapy, these studies provide insight into the IGF system in sarcomas and may pave the way to future therapeutic modalities. Given the early responses to the use of anti-IGF1R monoclonal antibodies and tyrosine kinase inhibitors in preclinical cancer models and in a few clinical studies such as Ewing sarcoma, a large number of companies produced these inhibitors and began largescale human cancer trials. Disappointingly, most of these trials failed. For example, a large trial on IGF1R inhibition in ER-positive breast cancer, cancers that usually express significant levels of IGF1Rs, showed lack of efficacy and some unexpected side effects. Yee (2018) further describes the rationale behind these trials, the disappointment with the lack of overall efficacy, reasons for the failures and direction for future therapies such as inhibiting the IGF1R in combination with other factors, such as the insulin receptor, which may compensate for the inhibition of the IGF1R. Conver \& Oxvig (2018) describe the role of pregnancy-associated plasma protein A (PAPPA) in cancer. PAPPA is a proteolytic enzyme that cleaves IGFBPs and allows bound IGFs to be free to interact with IGF1R on target tissue. They discuss the recent discovery that PAPPA is inhibited by stanniocalcins, what is currently known about PAPPA and stanniocalcins in different cancers. Interestingly, they propose that targeting PAPPA may be an alternative approach to inhibiting the IGF1 pathway in cancer.

The review by Gubbi et al. (2018) describes the role on IGF1 in aging, with a particular focus on the brain. Understanding its role is sometimes confusing and often seemingly contradictory. For example, in preclinical studies, IGF1 is important for protecting neurons by inhibiting apoptosis and possibly enhancing growth or differentiation. On the other hand, in Alzheimer's, reduced IGF1 levels may protect against the progression of disease. These seemingly conflicting results are similarly seen in clinical studies demonstrating both inverse correlations and direct correlations between circulating IGF1 levels and cognitive function. Additionally, in Parkinson's disease and cerebrovascular conditions, conflicting results have been described. Thus, while there are definite effects of high or low IGF1s on the brain, further research is definitely needed to understand these seemingly conflicting results.

Finally, Mohyi \& Smith (2018) describe his extensive research, culminating in the successful use of an anti-IGF1R monoclonal antibody in Graves' ophthalmopathy. Graves' disease is an autoimmune disorder where autoantibodies to the TSH receptor (TSHR) contribute to Graves' ophthalmopathy. The TSHR and IGF1R are known to interact and the anti-IGF1R monoclonal antibody demonstrated significant efficacy and safety in reducing the eye disease.

The issue editors are extremely excited by the compilation of these state-of-the-art reviews by world experts, as they cover the latest and most exciting aspects 
of ongoing IGF research, and we believe that readers will agree.

\section{Declaration of interest}

The authors declare that there is no conflict of interest that could be perceived as prejudicing the impartiality of this editorial.

\section{Funding}

This article did not receive any specific grant from any funding agency in the public, commercial or not-for-profit sector.

\section{References}

Bach LA 2018 IGF-binding proteins. Journal of Molecular Endocrinology 61 T11-T18. (https://doi.org/10.1530/JME-17-0254)

Clemmons D 2018 Role of IGF-binding proteins in regulating IGF responses to changes in metabolism. Journal of Molecular Endocrinology 61 T139-T169. (https://doi.org/10.1530/JME-18-0016)

Conover CA \& Oxvig C 2018 PAPP-A and cancer. Journal of Molecular Endocrinology 61 T1-T10. (https://doi.org/10.1530/JME-17-0236)

Gubbi S, Quipildor G F, Barzilai N, Huffman DM \& Milman S 2018 IGF-1: the Jekyll \& Hyde of the aging brain. Journal of Molecular
Endocrinology 61 T171-T185. (https://doi.org/10.1530/JME-180093)

Hakuno F \& Takahashi S-I 2018 IGF-I receptor signaling pathways. Journal of Molecular Endocrinology 61 T69-T86. (https://doi. org/10.1530/JME-17-0311)

Kineman RD, del Rio-Moreno M \& Sarmento-Cabral A 2018 Defining the tissue-specific roles of IGF1/IGF1R in regulating metabolism using the Cre/LoxP system. Journal of Molecular Endocrinology $\mathbf{6 1}$ T187-T198. (https://doi.org/10.1530/JME-18-0076)

Lindsey RC, Rundle CH \& Mohan S 2018 Role of IGF-I and Efn-Eph signaling in skeletal metabolism. Journal of Molecular Endocrinology 61 T87-T102. (https://doi.org/10.1530/JME-17-0284)

Mancarella C \& Scotlandi K 2018 IGF system in sarcomas: a crucial pathway with many unknowns to exploit for therapy. Journal of Molecular Endocrinology 61 T45-T60. (https://doi.org/10.1530/JME-170250)

Mohyi M \& Smith TJ 2018 IGF-I receptor and thyroid-associated ophthalmopathy. Journal of Molecular Endocrinology 61 T29-T43. (https://doi.org/10.1530/JME-17-0276)

Poinsot P, Schwarzer M, Peretti N \& Leulier F 2018 The emerging connections between IGF-1, the intestinal microbiome, Lactobacillus strains and bone growth. Journal of Molecular Endocrinology $6 \mathbf{1}$ T103-T113. (https://doi.org/10.1530/JME-17-0292)

Yakar S, Werner H \& Rosen CJ 2018 Insulin-like growth factors: actions on the skeleton. Journal of Molecular Endocrinology 61 T115-T137. (https://doi.org/10.1530/JME-17-0298)

Yee D 2018 Anti-insulin-like growth factor therapy in breast cancer Journal of Molecular Endocrinology 61 T61-T68. (https://doi. org/10.1530/JME-17-0261)

Received in final form 25 April 2018

Accepted 4 May 2018 